\title{
ESCAPE, ACCRETION, OR STAR FORMATION? THE COMPETING DEPLETERS OF GAS IN THE QUASAR MARKARIAN 231
}

\author{
Katherine Alatalo \\ Infrared Processing \& Analysis Center, California Institute of Technology, Pasadena, CA 91125, USA \\ Received 2014 December 27; accepted 2015 February 16; published 2015 March 3
}

\begin{abstract}
We report on high resolution $\mathrm{CO}(1-0), \mathrm{CS}(2-1)$, and $3 \mathrm{~mm}$ continuum Combined Array for Research in Millimeter Astronomy (CARMA) observations of the molecular outflow host and nearest quasar Markarian 231. We use the CS(2-1) measurements to derive a dense gas mass within Mrk 231 of $1.8 \pm 0.3 \times 10^{10} M_{\odot}$, consistent with previous measurements. The $\mathrm{CS}(2-1)$ data also seem to indicate that the molecular disk of Mrk 231 is forming stars at about normal efficiency. The high resolution CARMA observations were able to resolve the CO (1-0) outflow into two distinct lobes, allowing for a size estimate to be made and further constraining the molecular outflow dynamical time, further constraining the molecular gas escape rate. We find that $15 \%$ of the molecular gas within the Mrk 231 outflow actually exceeds the escape velocity in the central kiloparsec. Assuming that molecular gas is not constantly being accelerated, we find the depletion timescale of molecular gas in Mrk 231 to be $49 \mathrm{Myr}$, rather than $32 \mathrm{Myr}$, more consistent with the poststarburst stellar population observed in the system.
\end{abstract}

Key words: galaxies: active - galaxies: evolution - galaxies: ISM - galaxies: nuclei - quasars: individual (Markarian 231)

\section{INTRODUCTION}

Understanding the role of active galactic nuclei (AGNs) in their host galaxy's transition from a blue star-forming disk to a red quiescent ellipsoid is one of the forefront problems in studying galaxy evolution, with molecular gas at the center of the debate. A dearth of galaxies appearing in the optical "green valley" (Faber et al. 2007) seems to indicate that galaxies must transition between these two stages rapidly. It has been suggested that the expulsion of a galaxy's star-forming material through AGN feedback might provide a mechanism that is both sufficiently powerful and rapid to achieve this transition (Springel et al. 2005; Hopkins et al. 2006). Recent observations have shown many galaxies with AGNs host molecular outflows (Feruglio et al. 2010; Alatalo et al. 2011; Aalto et al. 2012b; Cicone et al. 2014; and references therein). In fact, AGN-driven molecular winds might be a ubiquitous property, as molecular observations of these types of objects become more numerous.

Markarian 231 (at a distance of $181 \mathrm{Mpc}$; Boksenberg et al. 1977) is an ideal laboratory to study the ways in which supermassive black holes and star formation interact with molecular gas. Mrk 231 has both a rapidly accreting, powerful AGN (Braito et al. 2004) and a starburst, with a star formation rate of $170 M_{\odot} \mathrm{yr}^{-1}$ (Veilleux et al. 2009), and is classified as an ultraluminous infrared galaxy (Sanders \& Mirabel 1996). Its disturbed morphology (Hutchings \& Neff 1987) confirms that it is in the late stages of an ongoing merger, which Hamilton \& Keel (1987) suggest is the origin of both the starburst and quasar activity. Recently, it has also been shown that Mrk 231 hosts a massive outflow, observed in molecular gas (Feruglio et al. 2010; Fischer et al. 2010; Aalto et al. 2012a), neutral gas (Rupke et al. 2005; Rupke \& Veilleux 2011; Teng et al. 2013), and warm ionized gas (Lipari et al. 2009; Rupke \& Veilleux 2011; Veilleux et al. 2013). These properties make Mrk 231 the ideal laboratory to test the ways in which dense molecular gas, star formation, and an AGN intermingle within a merging system, shedding light on the fate of the star-forming material, and ultimately, the galaxy as a whole, after a merger.
We present new high resolution Combined Array for Research in Millimeter Astronomy (CARMA; Bock et al. 2006) continuum, $\mathrm{CO}(1-0)$, and $\mathrm{CS}(2-1)$ observations of Mrk 231. In Section 2, we describe the observations and data analysis. In Section 3, we use the CARMA measurements to put the molecular gas in Mrk 231 into context, both in terms of star formation properties and depletion timescales. In Section 4, we present our conclusions. We use the cosmological parameters $H_{0}=70 \mathrm{~km} \mathrm{~s}^{-1}, \Omega_{M}=0.3$, and $\Lambda=0.7$ (Spergel et al. 2007) throughout.

\section{OBSERVATIONS AND DATA REDUCTION}

Mrk 231 was observed with CARMA between 2010 November and 2011 May in two different configurations: C array (1" resolution) and $\mathrm{B}$ array $(0, \cdot 7) .{ }^{12} \mathrm{CO}(1-0)$ and $\mathrm{CS}$ (2-1) were observed simultaneously, utilizing the upgraded correlator. The primary beam has a diameter of $2^{\prime}$ at $\mathrm{CO}(1-0)$, which covers all emission from Mrk 231. All observations used a long integration on a bright quasar to calibrate the passband, and alternated integrations between a gain calibrator (1419 +543 ) and Mrk 231. We then used the Mrk $2313 \mathrm{~mm}$ continuum source (Joyce et al. 1975) to self calibrate. The self calibration step was able to mitigate atmospheric fluctuations for the longer baseline arrays. The data were reduced using the Multichannel Image Reconstruction Image Analysis and Display (MIRIAD) software package (Sault et al. 1995). Calibration and data reduction steps were followed identically to Alatalo et al. (2013). Figure 1 presents the $\mathrm{CO}(1-0)$ spectrum, using the aperture defined by the integrated intensity (moment0) map (seen as an inset in Figure 1). Table 1 presents the derived properties from the CARMA observations.

The MIRIAD task uvlin was used to separate out continuum emission from Mrk 231 from the line emission, and we estimate the continuum flux of Mrk 231 to ${ }^{1}$ be $20.9 \pm 0.3 \mathrm{mJy}$ in the unself-calibrated data. A centroid was computed to determine

This does not include the absolute flux calibration uncertainty of $20 \%$. 


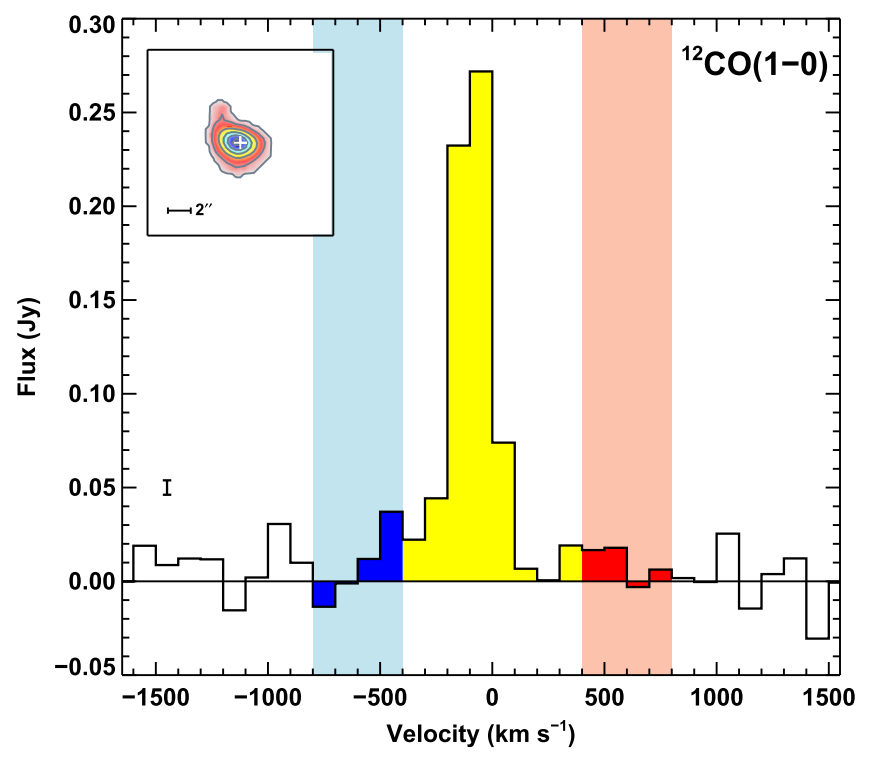

Figure 1. Integrated $\mathrm{CO}(1-0)$ spectrum of Mrk 231 from CARMA. The shaded regions correspond to the ranges summed to search for wing emission, of $400<v<800 \mathrm{~km} \mathrm{~s}^{-1}$ for the redshifted wing and $-800<v<-400$ $\mathrm{km} \mathrm{s}^{-1}$ for the blueshifted wing. The moment 0 map is shown as an inset in the top left, where a white cross pinpoints the location of the AGN.

the position of the $3 \mathrm{~mm}$ continuum and compare it to the VLBI-determined radio core (Ma et al. 1998), and found that the two positions agree to $0{ }^{\prime} \cdot 04$.

To image the blueshifted and redshifted wings, we created images with velocities between $-800<v<-400 \mathrm{~km} \mathrm{~s}^{-1}$ and $400<v<800 \mathrm{~km} \mathrm{~s}^{-1}$, respectively. Figure 1 shows the continuum-subtracted $\mathrm{CO}(1-0)$ spectrum derived from the $100 \mathrm{~km} \mathrm{~s}^{-1}$ CARMA channel maps, using the moment0 map (Figure 1 inset) as the masking aperture in each channel. The corresponding wing images and spectra are shown in Figure 2, overlaid on B-band Hubble Space Telescope imaging of Mrk 231 (right; Kim et al. 2013). CARMA derives a total flux of $74.3 \pm 3.0 \mathrm{Jy} \mathrm{km} \mathrm{s}^{-1}$, in agreement within errors with the flux recovered by Plateau de Bure (Feruglio et al. 2010; Cicone et al. 2012).

CARMA has successfully resolved the high velocity $\mathrm{CO}$ (1-0) wings, finding a separation between the centroids of 0 ". 49 (415 pc), a distance 5.8 times larger than the centroiding error, ${ }^{2}$ and the blue- and redshifted lobes have sizes of 2 !! 3 (1980 pc) and $2 ! 2(1820 \mathrm{pc})$, respectively. The fluxes associated with each imaged wing was determined by summing the emission that lay inside the $3 \sigma$ contours of the emission, and were found to be $F_{\mathrm{CO} \text {,blue }}=4.30 \pm 0.53 \mathrm{Jy} \mathrm{km} \mathrm{s}^{-1}$ and $F_{\mathrm{CO} \text {,red }}=4.60 \pm 0.53 \mathrm{Jy} \mathrm{km} \mathrm{s}^{-1}$, which is larger than what was found in Cicone et al. (2012), though it is possible that our apertures were larger. The lobes also appear to be separated in the east-west direction, with the centroid of the blue lobe located at $[12: 56: 14.245,+56: 52: 25.24]$ and for the red lobe centroid at [12:56:14.213, +56:52:25.21], with the $3 \mathrm{~mm}$ continuum point source (white cross) located between the lobes.

CS (2-1) observations are shown in Figure 3. Construction of channel maps, integrated intensity (moment0) maps, mean velocity (moment1) maps, spectra, and the corresponding determinations of the root mean square noise were performed

$\epsilon_{\text {centroid }}=\theta_{\text {beam }} /(2 \times \mathrm{SNR})$. identically to the methods described in Alatalo et al. (2013). CS(2-1) channels have a (optically defined) velocity width of $34 \mathrm{~km} \mathrm{~s}^{-1}$. The total $\mathrm{CS}(2-1)$ flux, determined by summing across the shaded channels in the spectrum, is $1.43 \pm 0.19 \mathrm{Jy} \mathrm{km} \mathrm{s}^{-1}$, and covers an area of $3.9 \operatorname{arcsec}^{2}$.

\section{RESULTS AND DISCUSSION}

\subsection{Dense Molecular Gas Traced by CS(2-1)}

Using the local thermal equilibrium $F_{\mathrm{CS}(2-1)}-M_{\mathrm{H}_{2} \text {,dense }}$ relation discussed in Alatalo et al. (2015) of

$$
N_{\mathrm{CS}}=1.90 \times 10^{11} T_{\mathrm{ex}} e^{7.05 / T_{\mathrm{ex}}} I_{\mathrm{CS}(2-1)},
$$

where $I_{\mathrm{CS}(2-1)}$ is the integrated intensity of the $\mathrm{CS}(2-1)$ line in $\mathrm{K} \mathrm{km} \mathrm{s}^{2-1}$, assuming $T_{\mathrm{ex}} \approx 70 \mathrm{~K}$ (van der Werf et al. 2010) and a CS abundance of $\approx 10^{-9}$, we find a total dense gas mass of $\approx 1.8 \pm 0.3 \times 10^{10} M_{\odot}$. This dense gas mass is consistent with the total dense gas mass found with other tracers (Solomon et al. 1992; Solomon \& Vanden Bout 2005; Feruglio et al. 2010), given the uncertainty in the $\mathrm{CS} / \mathrm{H}_{2}$ abundance, which is at least a factor of 2 . Our dense gas mass estimate is lower than the dynamical mass derived within $1100 \mathrm{pc}$ of the center $^{3}$ of Mrk 231 is $3.15 \times 10^{10} M_{\odot}$ (Downes \& Solomon 1998), necessitating that the majority of the central mass in Mrk 231 is in the form of dense molecular gas. The signalto-noise ratio of the CS data and resolution of these data are not able to confirm whether the CS is part of the face-on warped disk reported in Davies et al. (2004) and in dense gas reported in Aalto et al. (2015). Despite the limitation in resolution, the gradient seen in the CS moment1 map also appears consistent with the HCN kinematics seen by Aalto et al. (2012a). The agreement of the molecular gas mass also appears consistent with the idea that the majority of the molecular gas in Mrk 231 is in a dense form.

Our integrations were not sufficiently sensitive to detect CS (2-1) emission associated with broad wings (as is the case in other dense gas tracers, including $\mathrm{HCN}$, reported by Aalto et al. 2012a), and warrants deeper observations to determine whether gas traced by CS (suggested to be a tracer of some of the densest molecular cores; Baan et al. 2008) is also taking part in the molecular outflow.

To derive the dense gas column along the line of sight (LOS) to the AGN in Mrk 231 (white cross in Figure 3 moment maps), we find that the pixel associated with the radio point source contains an intensity of $51.8 \pm 2.0 \mathrm{~K} \mathrm{~km} \mathrm{~s}^{-1}$ (using a Kelvin per Jansky factor of 78.35). Converting this intensity into a column density of $\mathrm{H}_{2}$ (assuming an excitation temperature of $70 \mathrm{~K}$ and a $\mathrm{CS} / \mathrm{H}_{2}$ abundance of $10^{-9}$ ), we find a CS-derived LOS column to the AGN of $1 / 2 \times N_{H}=N\left(\mathrm{H}_{2}\right) \approx 1.1 \times 10^{24} \mathrm{~cm}^{-2}$ (if the AGN were sitting beneath the maximum value in the $\mathrm{CS}(2-1)$ map, the obscuring column would be $\left.N\left(\mathrm{H}_{2}\right) \approx 1.5 \times 10^{24} \mathrm{~cm}^{-2}\right)$. While this value was consistent with a previous estimate from X-ray absorption models (Braito et al. 2004), newer results from $N$ STAR show $N_{H}$ to be $1.2 \times 10^{23} \mathrm{~cm}^{-2}$ (Teng et al. 2014). The discrepancy between the CS-derived column might suggest that the obscuring medium in the nucleus of Mrk 231 is quite clumpy (at a scale much smaller than the CARMA resolution), which is consistent with the suggestion by Teng

\footnotetext{
3 Not including the uncertainty associated with converting CS to $M\left(\mathrm{H}_{2}\right)$ (Alatalo et al. 2015).
} 
Table 1

Mrk 231 Properties

\begin{tabular}{lccccccr}
\hline Tracer & $\begin{array}{c}\theta_{\text {maj }} \times \theta_{\text {min }} \\
\left({ }^{\prime \prime}\right)\end{array}$ & $\mathrm{K} \mathrm{per} \mathrm{Jy}^{\mathrm{a}}$ & $\begin{array}{c}\Delta v \\
\left(\mathrm{~km} \mathrm{~s}^{-1}\right)\end{array}$ & $\begin{array}{c}\text { rms } \\
\left(\mathrm{mJy} \mathrm{beam}^{-1}\right)\end{array}$ & $\begin{array}{c}\text { rms Per Channel } \\
(\mathrm{mJy})\end{array}$ & $\begin{array}{c}\text { Area } \\
\square^{\prime \prime}\end{array}$ & $\begin{array}{c}F_{\text {peak }} \\
\left(\mathrm{Jy} \mathrm{beam}^{-1} \mathrm{~km} \mathrm{~s}^{-1}\right)\end{array}$ \\
\hline $\mathrm{CO}(1-0)$ & $1.80 \times 1.57$ & 35.54 & 100 & 2.77 & 7.70 & 7.7 & $30.4 \pm 5.8$ \\
$\mathrm{CS}(2-1)$ & $1.40 \times 1.26$ & 78.35 & 34 & 1.28 & 1.82 & 3.9 & $0.92 \pm 0.13$ \\
\hline Blue & $1.19 \times 1.07$ & 78.48 & 400 & 0.57 & $\ldots$ & 5.3 & $1.91 \pm 0.3 \pm \pm 0.23$ \\
Red & $1.19 \times 1.07$ & 78.48 & 400 & 0.54 & $\ldots$ & 5.9 & $1.50 \pm 0.21$ \\
\hline
\end{tabular}

${ }^{a}$ Kelvin per Jansky factor for each observation.

et al. (2014) that the wind punching out of the system has created a preferential LOS to the AGN.

If we were to use the CS to investigate the star formation efficiency within Mrk 231, the total dense gas-traced surface density is $\Sigma_{\mathrm{H}_{2}} \approx 6450 M_{\odot} \mathrm{pc}^{-2}$. Comparing this to the star formation surface density (assuming the dense gas and star formation are co-spatial) of $61 M_{\odot} \mathrm{yr}^{-1} \mathrm{kpc}^{-2}$, we find that this matches the expectation from the Kennicutt-Schmidt relation (Kennicutt 1998).

\subsection{Resolved CO(1-0) Outflow}

Accounting for the distance to Mrk 231, 0."49 corresponds to a projected separation between the $\mathrm{CO}(1-0)$ centroids of $415 \mathrm{pc}$. The CARMA data also successfully resolve the lobes, with sizes of 2 !" 2 and 2 !" 3 of the red- and blueshifted wings, respectively. To deproject this distance, an accurate inclination angle is needed. Inclination angles derived for the molecular disk (which we assume is perpendicular to the outflow) has derived inclinations angles ranging from $i=10^{\circ}$ (Downes \& Solomon 1998; Davies et al. 2004) to $i=45^{\circ}$ (Richards et al. 2005), meaning that the deprojected distance ranges between 570 and $2390 \mathrm{pc}$. If we assume that the outflow is conical with a large opening angle (Rupke \& Veilleux 2011; Cicone et al. 2012), then the distance between the wings is likely similar to the diameter of the lobes, which we estimate to be $1800 \mathrm{pc}$ (after de-convolving the lobes). This size is slightly larger than the $\mathrm{CO}(1-0)$ separation found by Cicone et al. (2012), although we see extension in the east-west direction and opposite of the rotation of the CS disk, rather than the north-south direction as was observed in $\mathrm{HCN}$ by Aalto et al. (2012a). While this is consistent with the angle of rotation of the molecular disk, the velocities probed by the CARMA observations exceed the escape velocity, and therefore are unlikely to be due to rotation.

We use $600 \mathrm{~km} \mathrm{~s}^{-1}$ for the characteristic velocity of each wing (as well as the deprojected radius, $900 \mathrm{pc}$ ) to calculate the dynamical timescale of the outflow of $\tau_{\mathrm{dyn}} \approx 1.5 \mathrm{Myr}$, about twice as long as the timescale originally reported by Feruglio et al. (2010). If we use this dynamical timescale in conjunction with the outflow mass from Feruglio et al. (2010), and supported by Aalto et al. (2012a), of $5.8 \times 10^{8} M_{\odot}$, we find that the total mass outflow rate is $390 M_{\odot} \mathrm{yr}^{-1}$, a factor of 2 smaller than Feruglio et al. (2010), and a factor of $\approx 2$ larger than the star formation rate in the system (Veilleux et al. 2009). If all $390 M_{\odot} \mathrm{yr}^{-1}$ taking part in the molecular outflow were to escape the galaxy, we would expect the molecular gas to be depleted in $\approx 46 \mathrm{Myr}$ through the action of the molecular outflow alone, and $32 \mathrm{Myr}$ if both outflow and the star formation gas consumptions are considered. This depletion timescale assumes that all molecular gas in the thin disk is intercepted by the molecular outflow, which is assumed to be traveling perpendicular to the disk. If the molecular outflow is unable to interact with most of the gas in the molecular disk, this could increase the molecular outflow depletion timescale considerably.

\subsection{The Importance of Competing Depletion Mechanisms}

In Mrk 231, the molecular gas is being depleted by three competing mechanisms: consumption through star formation, escape from the galactic potential via a molecular outflow and accretion onto the supermassive black hole. The timescale for star formation to completely consume the central gas is $110 \mathrm{Myr}$. The molecular outflow appears to be the dominant depleter within the Mrk 231 system (assuming that the outflowing molecular mass is being completely expelled).

While $\dot{M}_{\text {out }}$ of Mrk 231, updated in the prior section is indeed larger than $\dot{M}_{\mathrm{SFR}}, \dot{M}_{\text {out }}$ might not be an accurate representation of the mass of molecular gas that is currently being expelled from the system. Mrk 231 is a massive system, with an estimated stellar mass of $\approx 3 \times 10^{11} M_{\odot}$ (U et al. 2012), and thus much of the outflowing molecular gas will not escape the potential well of Mrk 231, instead falling back and "stirring up" the massive molecular disk in the center. Using a dynamical mass within $1100 \mathrm{pc}$ of the center of Mrk 231 of $3.15 \times 10^{10} M_{\odot} \quad$ (Downes \& Solomon 1998; Davies et al. 2004), and derive an escape velocity from the central kiloparsec of $\sim 500 \mathrm{~km} \mathrm{~s}^{-1}$. If we assume that all gas that is traveling above $v_{\text {esc }}$ will be able to successfully escape the system, then $15 \%$ of the total outflowing molecular gas (from the Gaussian fit of Feruglio et al. 2010) escapes. ${ }^{4}$ We use our updated maps to calculate the total escaping mass from the blue- and redshifted wing fluxes reported in Section 2 and Table 1, using a conservative merger-based conversion factor (Narayanan et al. 2011), and find $M_{\mathrm{esc}}=(2.93 \pm 0.49) \times 10^{8} M_{\odot}$. Dividing by the dynamical time, we find $\dot{M}_{\text {esc }} \approx 195 M_{\odot} \mathrm{yr}^{-1}$, which is quite comparable to the current star formation rate of $170 M_{\odot} \mathrm{yr}^{-1}$ than were we to assume all outflowing mass is escaping. This updates the depletion timescale due to the escape of molecular gas, $\tau_{\mathrm{esc}}$, to $92 \mathrm{Myr}$. The combined depletion timescale $\tau_{\mathrm{SF}+\mathrm{esc}}$ is then 49 Myr.

The accretion onto the black hole can be derived using the bolometric luminosity of the AGN $\left(\approx 2.8 \times 10^{12} L_{\odot}\right.$; Veilleux et al. 2009, 2013), and assuming an efficiency $\eta=0.17$, to be $\dot{M}_{\text {acc }} \approx 1 M_{\odot} \mathrm{yr}^{-1}$, or at a rate $\sim 10^{-2}$ other depletion rates, a

\footnotetext{
4 This estimate only accounts for the the molecular gas traced by $\mathrm{CO}(1-0)$, although the Mrk 231 outflow has been detected in additional tracers of netural gas, including $\mathrm{OH}$ (Sturm et al. 2011), Na D (Veilleux et al. 2009), and $\mathrm{H}_{1}$ (Teng et al. 2013).
} 

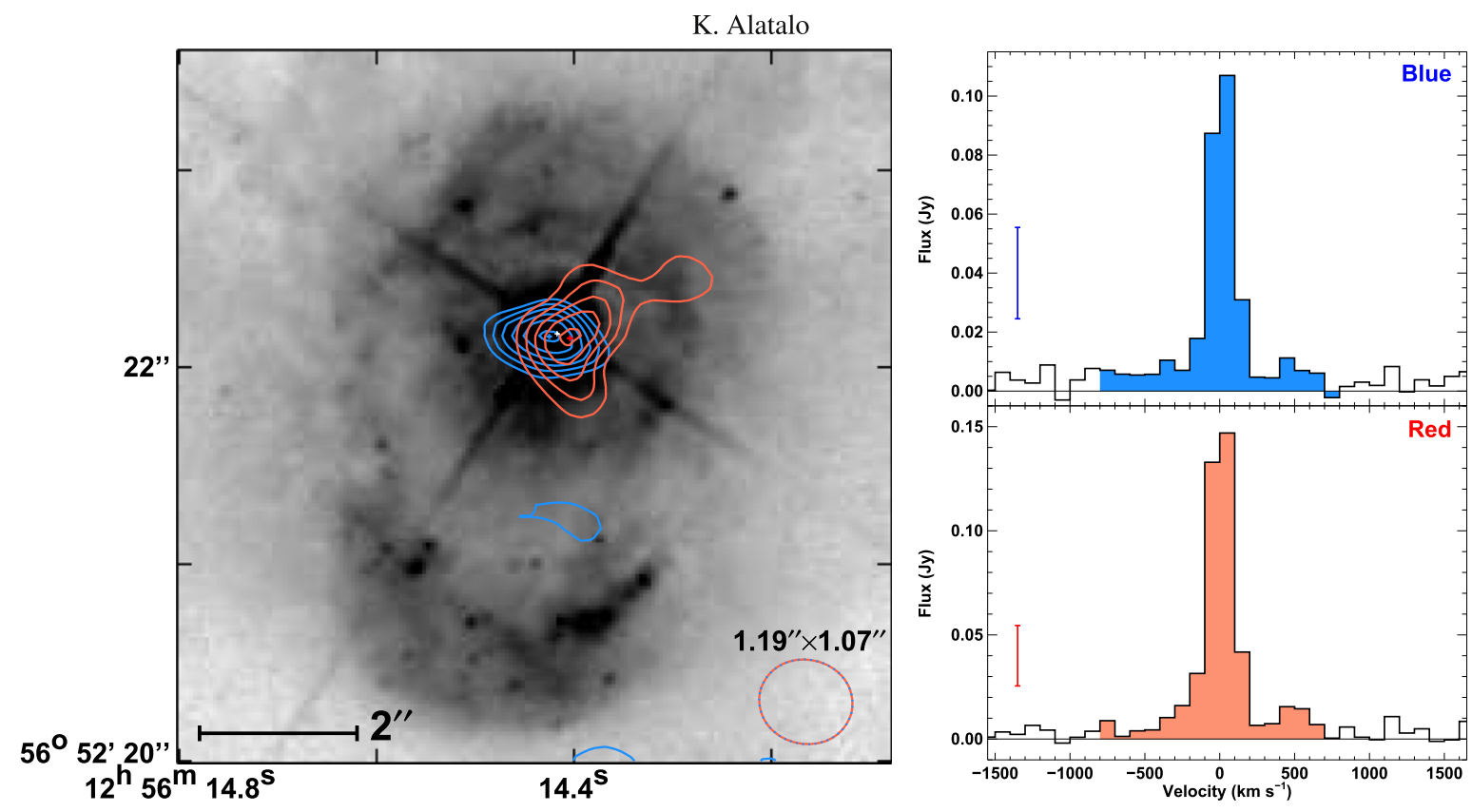

Figure 2. (Left) $B$-band archival image of Mrk 231 from $H S T$ (grayscale; Kim et al. 2013) is overlaid with the integrated CARMA maps of the blueshifted $\left(-800<v<-400 \mathrm{~km} \mathrm{~s}^{-1}\right.$; blue $)$ and redshifted $\left(400<v<800 \mathrm{~km} \mathrm{~s}^{-1}\right.$; red) wings. Contours for the red lobe is $[3,4,5,6,7] \sigma$ and $[3,4,5,6,7,8] \sigma$ for the blue lobe, where $\sigma$ is the root mean square noise of the map. The blue and red crosses represent the centroids on the blue- and redshifted wings, respectively. The white cross represents the position of the centroid of the $3 \mathrm{~mm}$ continuum point source. Separation between the lobes is $\approx 0 . " 49$, a well-resolved distance, where the size of the red and blue crosses represent the centroiding errors for each wing. The separation is detected to 5.8 times the centroiding error. It is of note that the $\mathrm{CO}(1-0)$ wings velocity range almost entirely exceeds the escape velocity out of the central kiloparsec of Mrk 231. (Right) Integrated $\mathrm{CO}(1-0)$ spectrum of Mrk 231 using emission exceeding a signal-to-noise ratio of three in red- and blueshifted wing images to create the extraction apertures. This seems to indicate that CARMA has successfully detected emission in the red- and blueshifted wings.

minor constituent to the depletion of the central molecular gas, and requiring a Hubble time to completely remove the molecular gas from the system.

Without a constant driving mechanism, the majority of the molecular outflow will eventually fall back into the center, either to be accreted, formed into stars, or re-launched. One would expect that this gas would fall back and re-inject energy into the central molecular disk, possibly acting to inhibit star formation (Alatalo et al. 2015; Guillard et al. 2015) and therefore extend the molecular gas consumption timescale. An extension of the depletion timescale expected for Mrk 231 from $\sim 20 \mathrm{Myr}$ (the typical lifetime for O-stars) to $\sim 50 \mathrm{Myr}$, appears more consistent with the poststarburst population found in Mrk 231 (Canalizo \& Stockton 2000), and common in other quasars (Cales et al. 2011; Canalizo \& Stockton 2013), as well as other hosts of molecular outflows (such as NGC 1266; Alatalo et al. 2011, 2014).

The rapidity at which Mrk 231 is currently expelling its molecular material compared with weaker AGNs might be due to the different circumstances that created this system and outflow. A major merger occurred in a system already sufficiently massive to host a massive black hole, which is thus energetically capable of driving the bulk of the molecular gas from the system. Sub-vesc gas would be able to fall back into the system and inject turbulence, in effect temporarily stalling efficient star formation and possibly extending the depletion time just slightly. This would account for the mismatch in depletion timescales between $\sim 30 \mathrm{Myr}$ (the depletion timescale should all outflowing gas escape and with the current star formation rate) and $\sim 50 \mathrm{Myr}$ (the stellar population age seen in Mrk 231; Canalizo \& Stockton 2000).
Deeper observations of the molecular outflow in Mrk 231, focusing on spatial resolution on at different distances away from the AGN will be able to determine what fraction of the molecular gas is escaping the gravitational potential, and provide a more complete understanding of the driving mechanism as well as the energy injection rate. Low frequency imaging could pinpoint the location of fossil shells (Schoenmakers et al. 2000), as Mrk 231 has already been detected at low frequency (Cohen et al. 2007). Combining these observations with the multiwavelength suite available for Mrk 231 will provide additional constraints, such as whether the molecular outflow changes phase as it accelerated, and whether there is any evidence that gas ultimately falls back into the molecular disk of Mrk 231.

\section{CONCLUSIONS}

We have presented $3 \mathrm{~mm}$ continuum and $\mathrm{CO}(1-0)$ and $\mathrm{CS}$ (2-1) high resolution molecular gas maps of Mrk 231 from CARMA. The CS(2-1) data paint a picture of the dense molecular gas in Mrk 231 that is consistent with other dense gas tracers (Aalto et al. 2012a). CS(2-1) predicts a dense gas mass $\left(\right.$ of $\left.M_{\text {dense }}=1.8 \pm 0.3 \times 10^{10} M_{\odot}\right)$ and an LOS column toward the AGN of $N\left(\mathrm{H}_{2}\right) \approx 10^{24} \mathrm{~cm}^{-2}$, inconsistent with the X-ray derived column (Teng et al. 2014), possibly indicative of a clumpy ISM and the AGN-driven winds punching a preferential LOS to the AGN. If we use the dense gas to derive the star formation efficiency, we find that Mrk 231 is consistent with the prediction of the Kennicutt-Schmidt relation.

CARMA was able to resolve the individual $\mathrm{CO}(1-0)$ broad line wings, with an inferred separation between the wings to be 

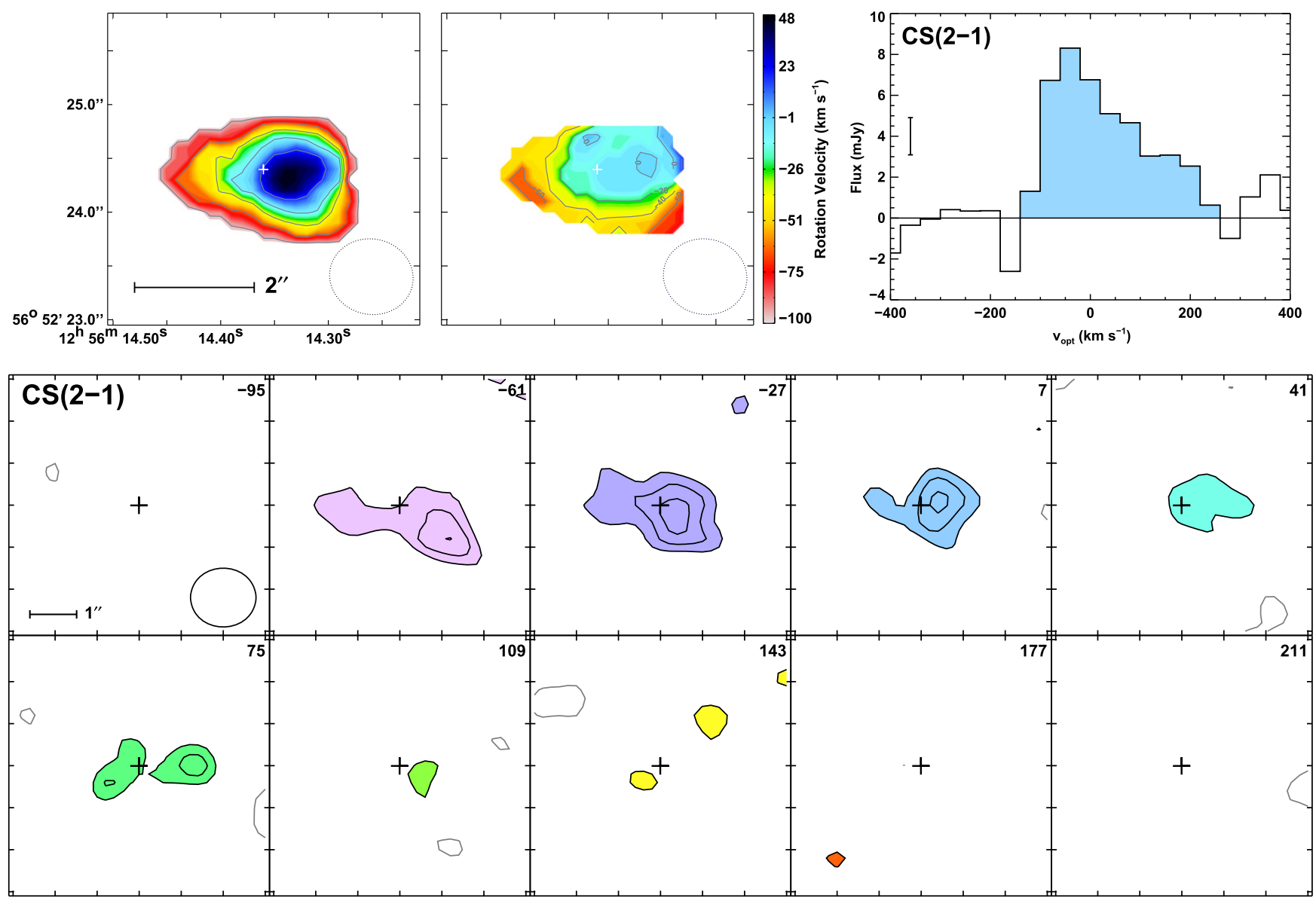

Figure 3. (Top left and middle) Integrated intensity (moment0) and mean velocity (moment1) maps of CS(2-1) within Mrk 231 from CARMA. While there does appear to be some ordered motion, overall the spectrum appears quite turbulent. Contour levels are $[0.01,0.1,0.3,0.5,0.7,0.9]$ times the maximum value of the moment0 map. (Top right) Integrated CS(2-1) spectrum, with the rms per channel shown as a small error bar on the left-hand side of the spectrum. The CS(2-1) line appears to have a width of $\approx 300 \mathrm{~km} \mathrm{~s}^{-1}$. (Bottom) $\mathrm{CS}(2-1)$ channel maps. Contours are $[-2.5,2.5,3.5,4.5] \sigma$, where $\sigma$ is the rRMS of the CS(2-1) cube. The negative contours are gray.

$\approx 1.8 \mathrm{kpc}$ given the angular size of the lobes and possible inclination angles of the outflow. This is slightly larger than the $\mathrm{CO}(1-0)$ lobe separation reported in Cicone et al. (2012). If we assume that not all molecular gas will successfully escape the galaxy, we can re-calculate the depletion timescale for Mrk 231 based on the mass escape rate of $\approx 195 M_{\odot} \mathrm{yr}^{-1}$. A depletion timescale using $\dot{M}_{\text {esc }}+\dot{M}_{\text {SFR }}$ rather than $\dot{M}_{\text {out }}+\dot{M}_{\text {SFR }}$ is more consistent with the poststarburst stellar populations that are seen in Mrk 231 as well as many other quasars.

K.A. would like to thank Mark Lacy, Lauranne Lanz, and Kristina Nyland for useful conversations and proofreading, which improved the text, as well as the anonymous referee, for a substantive report that has significantly improved the manuscript. K.A. is supported by funding through Herschel, a European Space Agency Cornerstone Mission with significant participation by NASA, through an award issued by JPL/ Caltech. Support for CARMA construction was derived from the states of California, Illinois, and Maryland; the Gordon and Betty Moore Foundation; the Kenneth T. and Eileen L. Norris Foundation; the Associates of the California Institute of Technology; and the National Science Foundation. Ongoing CARMA development and operations are supported by the National Science Foundation under a cooperative agreement, and by the CARMA partner universities. This research has made use of the NASA/IPAC Extragalactic Database (NED) which is operated by the Jet Propulsion Laboratory, California Institute of Technology, under contract with the National Aeronautics and Space Administration. Based on observations made with the NASA/ESA Hubble Space Telescope, and obtained from the Hubble Legacy Archive, which is a collaboration between the Space Telescope Science Institute (STScI/NASA), the Space Telescope European Coordinating Facility (ST-ECF/ESA) and the Canadian Astronomy Data Centre (CADC/NRC/CSA).

\section{REFERENCES}

Aalto, S., Garcia-Burillo, S., Muller, S., et al. 2012a, A\&A, 537, A44 Aalto, S., Garcia-Burillo, S., Muller, S., et al. 2015, A\&A, 574, A85 Aalto, S., Muller, S., Sakamoto, K., et al. 2012b, A\&A, 546, A68 Alatalo, K., Blitz, L., Young, L. M., et al. 2011, ApJ, 735, 88

Alatalo, K., Davis, T. A., Bureau, M., et al. 2013, MNRAS, 432, 1796

Alatalo, K., Lacy, M., Lanz, L., et al. 2015, ApJ, 798, 31

Alatalo, K., Nyland, K., Graves, G., et al. 2014, ApJ, 780, 186

Baan, W. A., Henkel, C., Loenen, A. F., Baudry, A., \& Wiklind, T. 2008, A\&A, 477, 747

Bock, D. C.-J., Bolatto, A. D., Hawkins, D. W., et al. 2006, Proc. SPIE, 6267, 626713

Boksenberg, A., Carswell, R. F., Allen, D. A., et al. 1977, MNRAS, 178, 451

Braito, V., della Ceca, R., Piconcelli, E., et al. 2004, A\&A, 420, 79

Cales, S. L., Brotherton, M. S., Shang, Z., et al. 2011, ApJ, 741, 106

Canalizo, G., \& Stockton, A. 2000, AJ, 120, 1750 
Canalizo, G., \& Stockton, A. 2013, ApJ, 772, 132

Cicone, C., Feruglio, C., Maiolino, R., et al. 2012, A\&A, 543, A99

Cicone, C., Maiolino, R., Sturm, E., et al. 2014, A\&A, 562, A21

Cohen, A. S., Lane, W. M., Cotton, W. D., et al. 2007, AJ, 134, 1245

Davies, R. I., Tacconi, L. J., \& Genzel, R. 2004, ApJ, 613, 781

Downes, D., \& Solomon, P. M. 1998, ApJ, 507, 615

Faber, S. M., Willmer, C. N. A., Wolf, C., et al. 2007, ApJ, 665, 265

Feruglio, C., Maiolino, R., Piconcelli, E., et al. 2010, A\&A, 518, L155

Fischer, J., Sturm, E., González-Alfonso, E., et al. 2010, A\&A, 518, L41

Guillard, P., Boulanger, F., Lehnert, M. D., et al. 2015, A\&A, 574, A32

Hamilton, D., \& Keel, W. C. 1987, ApJ, 321, 211

Hopkins, P. F., Hernquist, L., Cox, T. J., et al. 2006, ApJS, 163, 1

Hutchings, J. B., \& Neff, S. G. 1987, AJ, 93, 14

Joyce, R. R., Knacke, R. F., Simon, M., \& Young, E. 1975, PASP, 87, 683

Kennicutt, R. C., Jr. 1998, ApJ, 498, 541

Kim, D.-C., Evans, A. S., Vavilkin, T., et al. 2013, ApJ, 768, 102

Lipari, S., Sanchez, S. F., Bergmann, M., et al. 2009, MNRAS, 392, 1295

Ma, C., Arias, E. F., Eubanks, T. M., et al. 1998, AJ, 116, 516

Narayanan, D., Krumholz, M., Ostriker, E. C., \& Hernquist, L. 2011, MNRAS, 418,664
Richards, A. M. S., Knapen, J. H., Yates, J. A., et al. 2005, MNRAS, 364, 353 Rupke, D. S., Veilleux, S., \& Sanders, D. B. 2005, ApJS, 160, 115

Rupke, D. S. N., \& Veilleux, S. 2011, ApJL, 729, L27

Sanders, D. B., \& Mirabel, I. F. 1996, ARA\&A, 34, 749

Sault, R. J., Teuben, P. J., \& Wright, M. C. H. 1995, in ASP Conf. Ser. 77, Astronomical Data Analysis Software and Systems IV, ed. R. A. Shaw, H. E. Payne, \& J. J. E. Hayes (San Francisco, CA: ASP), 433

Schoenmakers, A. P., de Bruyn, A. G., Röttgering, H. J. A. van der Laan, H., \& Kaiser, C. R. 2000, MNRAS, 315, 371

Solomon, P. M., Downes, D., \& Radford, S. J. E. 1992, ApJL, 387, L55

Solomon, P. M., \& Vanden Bout, P. A. 2005, ARA\&A, 43, 677

Spergel, D. N., Bean, R., Doré, O., et al. 2007, ApJS, 170, 377

Springel, V., di Matteo, T., \& Hernquist, L. 2005, ApJL, 620, L79

Sturm, E., González-Alfonso, E., Veilleux, S., et al. 2011, ApJL, 733, L16

Teng, S. H., Brandt, W. N., Harrison, F. A., et al. 2014, ApJ, 785, 19

Teng, S. H., Veilleux, S., \& Baker, A. J. 2013, ApJ, 765, 95

U, V., Sanders, D. B., Mazzarella, J. M., et al. 2012, ApJS, 203, 9

van der Werf, P. P., Isaak, K. G., Meijerink, R., et al. 2010, A\&A, 518, L42

Veilleux, S., Rupke, D. S. N., Kim, D.-C., et al. 2009, ApJS, 182, 628

Veilleux, S., Trippe, M., Hamann, F., et al. 2013, ApJ, 764, 15 